\title{
EXPERIMENTAL AND NUMERICAL STUDY OF THE HEAT TRANSFER AND PRESSURE DROP IN TRIANGULAR CHEVRON CHANNELS
}

\author{
Hossein Dolatabadi, Abolfazl Hajizadeh Aghdam \\ Department of Mechanical Engineering, Arak University of Technology, Arak, Iran \\ e-mail: abolfazl_hajizade@yahoo.com
}

\begin{abstract}
Chevron channels are one of the popular techniques that are extensively used in manufacturing of compacted heat exchangers. The present paper deals with the experimental and numerical analysis on the fluid flow and heat transfer in a triangular chevron channel. The studies are carried out for a uniform wall heat flux equal to $1350 \mathrm{~W} / \mathrm{m}^{2}$ using air as the working fluid. The Reynolds number varies from 1000 to 10000 , phase shifts $0^{\circ} \leqslant \phi \leqslant 180^{\circ}$, and channel heights are $5 \leqslant D \leqslant 35 \mathrm{~mm}$. The study shows a significant effect of the optimum structure of the triangular chevron on the heat transfer rate and friction loss over the channel wall. The Nusselt number increases and the Thermal Enhancement Factor (TEF) decreases with the increasing Reynolds number. The best performance is noticed on the phase shift, $\phi=90^{\circ}$. For triangular chevron surfaces, the maximum heat transfer is obtained $33.33 \%$ up to $37.5 \%$ more than for a smooth wall channel.
\end{abstract}

Keywords: heat transfer, pressure drop, thermal enhancement factor, phase shift

\section{Introduction}

Chevron channels are basic channel geometry in plate heat exchangers because of their efficient heat exchange capabilities. Today, to increase the heat flux rate in nuclear reactors, turbine blades and electronic equipment, some companies are using chevron surfaces. Chevron surfaces create turbulent sublayer of laminar flows, as a result the heat transfer becomes increased. When the friction factor increases, the fan power will increase for a stable velocity of the fluid flow. To generate more power and enhance system performance of designed turbines, the inlet temperature of the fluid must be increased. In some cases high temperature of the fluid increases the temperature of turbine blades to the melting point. In such situations making use of alloy or super alloys might be a technical solution, but it is justified ecconomically. To reduce temperature of hot surfaces, one has to use surfaces with spatial geometry including ribs or chevron surfaces. The experience proves that chevron surfaces lead to a suitable pressure drop. In solar systems, the fluid flow is laminar so the heat transfer is low, and the chevron surfaces create turbulent sublayers increasing the heat transfer. One can increase the heat transfer in fluids by: $1-$ separation of fluids, 2 - making laminar sublayers turbulent, 3 - replacing flows in the hot surface. The first major problem in designing heat exchanger is to save more energy and increase the heat trasfer rate as well as reduce friction. Thus, chevron surfaces play an important here.

The flow and heat transfer in chevron channels have been extensively investigated in the recent years, see for example Yang and Chen (2010), Vanaki et al. (2014), Sakr (2015).

Khoshvaght-Aliabadi et al. (2016) found a significant improvement in the heat transfer coefficient by using pure water instead of a water-ethylene glycol mixture. However, the Nusselt number increased considerably with a grow in the weight percentage of ethylene glycol in the working fluid. The convective thermal resistance was noticeably reduced by using the SWMCHSs. As an example, a reduction of $113.8 \%$ was obtained for the water flow at the mass flow rate of 
$0.024 \mathrm{~kg} / \mathrm{s}$ in the SWMCHS with $l=20 \mathrm{~mm}$ and $a=1.0 \mathrm{~mm}$, compared to the straight MCHS at the same conditions.

Yongsiri et al. (2014) found that the ribs which induced recirculation gave a higher Nusselt number and friction factor than those which did not. Among the ribs examined, the ones with $\theta=60$ yielded a comparable heat transfer rate 1.74 times of those in the smooth channel, and $\theta=120$ yielded a thermal performance factor 1.21 which was higher than those given by others.

Al-Shamani et al. (2015) showed interesting results of changing rib groove shapes in thermal and fluid fields. The results proved that trapezoidal grooves with increasing height in the flow direction (Trap+R-TrapG) gave the highest Nusselt number in comparison with other types of trapezoidal channels. 1) By changing types of nano particles $\left(\mathrm{Al}_{2} \mathrm{O}_{3}, \mathrm{CuO}, \mathrm{SiO}_{2}\right.$, and $\left.\mathrm{ZnO}\right)$ the results revealed that $\mathrm{SiO}_{2}$ led to the highest Nusselt number, then followed by $\mathrm{Al}_{2} \mathrm{O}_{3}, \mathrm{ZnO}$, and $\mathrm{CuO}$, respectively, while pure water gave the lowest value of Nusselt number. 2) The Nusselt number increased with the increasing volume fraction of nanoparticles. 3) The Nusselt number increased gradually with a decrease in diameter of the nano particles. 4) The Nusselt number increased gradually with growth in the Reynolds number in the range of 10 000-40000.

Eiamsa-ard and Changcharoen (2011) found that by reducing the degree of sudden changes of the main flow, the flow separation suppresses and thus the corner separation of bubbles in front and rear surfaces of the rib decreases. The streamlines proved that among the ribs with concave surfaces unrecognized corner separation of bubbles appeared, whereas those with modified convex surfaces generated corner separation of bubbles with size comparable to that of the unmodified square rib. All ribs with concave surfaces induced a larger recirculation zone than the others, resulting in high turbulence intensity. With good conformation of the streamlines, the rib with concave-concave surfaces gave the highest Nusselt number and the friction factor while those with convex-concave surfaces provided the lowest friction factor with moderate Nusselt numbers.

Due to the prominent effect of a low friction factor, the rib with the convex-concave surface offers the highest TEF with the maximum value of 1.19 .

Xie et al. (2014) studied the flow structure and heat transfer in a square passage with offset mid-truncated ribs.

The heated surface of $135^{\circ} \mathrm{V}$-shaped mid-truncated ribs provided the highest heat transfer enhancement, while the heated surface of $90^{\circ}$ mid-truncated ribs with no staggered arrangement behaved best in reducing the pressure loss penalty.

Moon et al. (2014) proved that the new boot-shaped rib gave the best heat transfer performance with an average friction loss performance, and the reverse pentagonal rib gave the best friction loss performance.

Dellil et al. (2004) conducted a geometrical parametric study by changing the amplitudeto-wavelength ratio. Comparison of predicted results for a wavy wall with those for a straight channel indicated that the averaged Nusselt number increased until a critical value was reached where the amplitude wave was increased. However, that heat transfer enhancement was accompanied by an increase in the pressure drop.

Characteristics of the fluid flow and heat transfer in a periodic fully developed region of a corrugated duct were numerically obtained using the finite element software by Islamoglu and Parmaksizoglu (2004). Both the heat transfer coefficient and the pressure drop for the corrugated ducts were in good agreement. In addition, the finite element technique can be used to simulate heat exchanger channels.

However, the present study is concerned with a special triangular chevron channel with the focus on variation in the phase shift $\phi$ and distance of plates $D$. The experimental and numerical methods have been used to find the best $\phi$ and $D$ with considering the maximum $\mathrm{Nu}$ and TEF as well as the minimum friction factor. 


\section{Experimental setup}

The experimental set up is shown in Fig. 1. The plate geometry is $125 \times 80 \mathrm{~mm}$. A forced-convection air flow was used in the experiments and an equal power input for heated length was established in the bottom walls. To measure the temperature distribution of each chevron wall, nine thermocouples (KTJ model TA-288) were used with $4 \mathrm{~mm}$ diameter holes drilled into the side wall (number 8 in Fig. 1). These holes were located at axial distances of (T1), (T2), (T3), (T4), (T5), (T6), (T7), (T8), (T9). The accuracy of the thermocouples was $\pm 1^{\circ} \mathrm{C}$. A U-manometer was located at the chevron surface to measure pressure drop within the triangular chevron channel (number 4 in Fig. 1). The accuracy of pressure transducer was $\pm 1 \mathrm{~mm}_{2} \mathrm{H}_{6} \mathrm{O}$. The test section was also isolated to avoid thermal losses. The experimental procedure involved adjusting the flow rate to the desired value (number 1 in Fig. 1). After the fan was turned on and the desired Reynolds number was obtained, the power input of plate heaters (number 6 in Fig. 1) gradually increased and maintained at $1350 \mathrm{~W} / \mathrm{m}^{2}$ to provide sufficient measurement while the fluid property varied. The heat supplied into the chevron walls was adjusted to achieve the desired level by using electric heaters, which were $1.314 \mathrm{~mm}$ thick, $80 \mathrm{~mm}$ wide and $150 \mathrm{~mm}$ long. They were located at the back of the bottom triangular chevron plate. The voltage and current of the electric input to the plate type heaters were controlled by a DC power supply (P.A. Hilton model Ltd H111/07332) unit (number 5 in Fig. ). Temperatures were recorded at intervals of 15 min until a steady state was reached. Steady state conditions were assumed to prevail when temperature measurements (number 8 in Fig. 1) on the plates were within $\pm 1^{\circ} \mathrm{C}$.

These instruments were placed and fitted in a container made of the plexiglas insulator. Figure 1 shows the assembled configuration of the test module with the fluid entrance and exit from the channel. The chevron surfaces are fabricated from blocks of aluminum 1060 alloy whose physical properties are shown in Table 1.

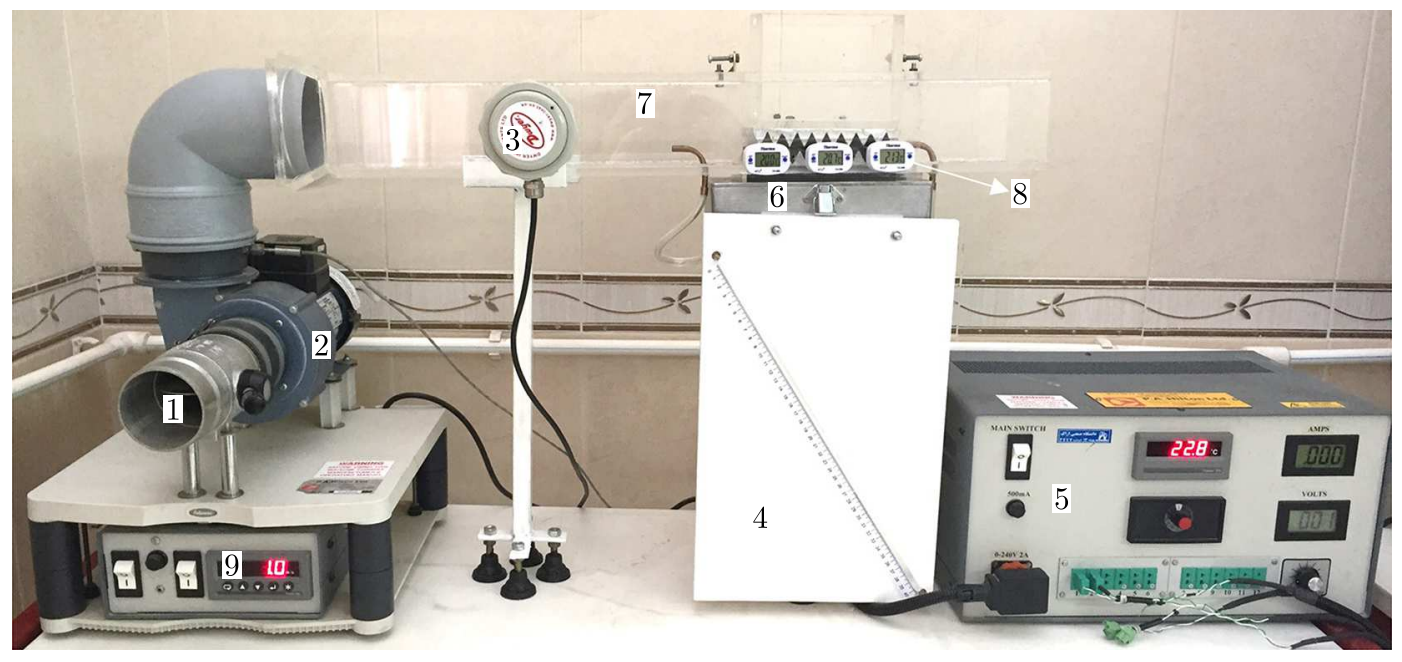

Fig. 1. Assembled configuration of the test module with triangular chevron surfaces

A centrifugal fan which was made of stainless steel and had the inner diameter of $90 \mathrm{~mm}$ (number 2 in Fig. 1) sucked the room air at the room temperature and exhausted into the atmosphere through the test section (that made of plexiglass with $8 \mathrm{~mm}$ thickness (number 7 in Fig. 1)). The flow was controlled by a control valve placed inside the fan (number 1 in Fig. 1) and the air velocity was calculated by a velocity sensor (Dwyer AVU-3V model HP111 LH) as shown in Fig. 1 (number 3). The display on the DC power controller of the centrifugal fan (P.A. Hilton Ltd H111/00629) is shown (number 9) in Fig. 1. The accuracy of the velocity sensor was $\pm 0.1 \mathrm{~m} / \mathrm{s}$. 
Table 1. Physical properties of the aluminum 1060 alloy

\begin{tabular}{|l|c|c|}
\hline \multicolumn{2}{|c|}{ Properties } & Conditions \\
\cline { 3 - 3 } & $T\left[{ }^{\circ} \mathrm{C}\right]$ \\
\hline \hline Density $\left[\times 1000 \mathrm{~kg} / \mathrm{m}^{3}\right]$ & 2.7 & 25 \\
\hline Poisson's ratio & 0.33 & 25 \\
\hline Elastic modulus $[\mathrm{GPa}]$ & $70-80$ & 25 \\
\hline Hardness (HB500) & 23 & 25 \\
\hline Thermal expansion & $23.610^{-6} /{ }^{\circ} \mathrm{C}$ & $20-100$ \\
\hline Thermal conductivity & $234 \mathrm{~W} / \mathrm{mK}$ & 25 \\
\hline
\end{tabular}

\subsection{Physical model}

Geometry of the experimental setup of the channel is shown in Fig. 2. The physical properties of the air have been assumed to remain constant at the average bulk temperature. Impermeable boundary and no-slip wall conditions have been assumed over the channel walls as well as the chevron surfaces. The distance between the apexes of the triangle with the vertical axis coordinates $\left(D_{v}=7.5 \mathrm{~mm}\right)$ was constant. Length of the channel in front of the test section was $L_{\text {inlet }}=30 \mathrm{~cm}$ and length of the channel behind the test section $L_{\text {outlet }}=25 \mathrm{~cm}$. Length of the test section was $\left(L_{\text {test }}\right) 15 \mathrm{~cm}$ and height and length of the ribs were $H=L=15 \mathrm{~mm}$. Location of thermocouples shown with a blue circle was $H_{T}=7.5 \mathrm{~mm}$ of the base chevron surface.

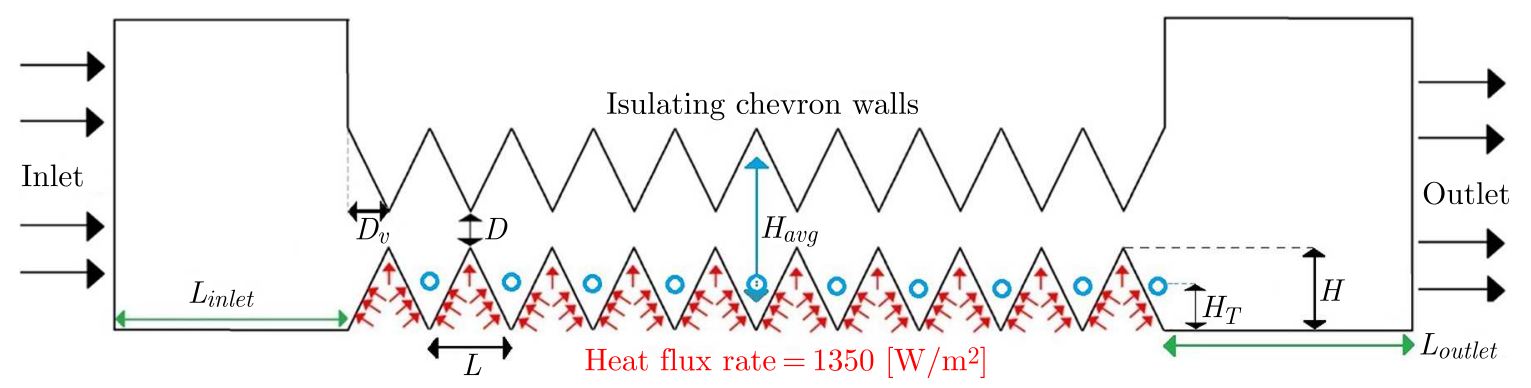

Fig. 2. Geometry of the experimental setup of the channel

The next section creates the entering boundary condition with a heat flux rate of the heater $1350 \mathrm{w} / \mathrm{m}^{2}$, The other wall is an insulating one and the inlet section with the input speed (velocity inlet) as well as the fluid outlet (pressure outlet). The inlet temperature of the working fluid (air) was kept constant at $298.15 \mathrm{~K}$.

\section{Performance parameters}

Parameters of interest in the present work are the Reynolds number, friction factor, Nusselt number and hydraulic diameter

$$
D_{h}=\frac{2 H_{\text {avg }} W}{H_{\text {avg }}+W}
$$

where $H_{\text {avg }}$ is average distance between the chevron surfaces, and $W$ is width of the chevron surfaces.

$$
\operatorname{Re}=\frac{\rho u D_{h}}{\mu}
$$


where $u$ is velocity of the fluid at the inlet of the test section, $D_{h}$ is hydraulic diameter and $\mu$ is dynamic viscosity

$$
f=2 \frac{\Delta p}{L}\left(\frac{D_{h}}{\rho u^{2}}\right)
$$

The friction factor is computed by the pressure drop $\Delta P$ across length of the duct $L$, and $\rho$ is density of flow.

$$
h_{x}=\frac{\ddot{q}}{T_{x}-T_{b}}
$$

where $h_{x}$ is the local convective heat transfer coefficient, $T_{x}$ is the local temperature, $T_{b}$ is the bulk temperature and $\ddot{q}$ is heat flux. The heat transfer was measured through the local Nusselt number which can be written as

$$
\mathrm{Nu}_{x}=\frac{h_{x} D_{h}}{k}
$$

where $\mathrm{Nu}$ is the Nusselt number and $K$ is conductivity. Then, the average Nusselt number can be obtained by

$$
\mathrm{Nu}=\frac{1}{L} \int \mathrm{Nu}_{x} d x
$$

and

$$
\mathrm{Nu}_{s}=0.024 \operatorname{Re}^{0.8} \operatorname{Pr}^{0.4} \quad f_{s}=0.085 \operatorname{Re}^{-0.25}
$$

where $\mathrm{Nu}_{s}$ and $f_{s}$ stand respectively for the Nusselt number and friction factor of the smooth duct. The Thermal Enhancement Factor (TEF) can be written according to Eiamsa-ard and Changcharoen (2011)

$$
\mathrm{TEF}=\frac{\mathrm{Nu}}{\mathrm{Nu}_{s}}\left(\frac{f}{f_{s}}\right)^{-\frac{1}{3}}
$$

\section{Numerical model}

Numerical analysis of the thermal behavior and flow dynamic characteristics of the chevron channel has been carried out to predict the heat transfer and pressure drop. The governing equations have been solved using a finite volume approach. The time-independent incompressible Navier-Stokes equations and the turbulence model were discretized using the finite volume method. Many investigators predicted turbulent forced convection in a rectangular duct with periodic chevron shapes by utilizing different turbulence models, such as $k-\varepsilon, k-\omega$, Reynolds stress model $(k-\varepsilon)$ and large eddy simulations (LES) models. The $k-\varepsilon$ model made from the two-equation models when predicting flow patterns of revolving flows.

In the present study, the $k-\varepsilon$ model is used for the turbulence modeling, and the SIMPLE algorithm is used to handle the pressure-velocity coupling. The discretized nonlinear equations are implemented implicitly. To evaluate the pressure field, the pressure-velocity coupling algorithm SIMPLE (Semi Implicit Method for Pressure-Linked Equations) is selected. The following assumptions are applied in the simulations: the flow is steady, fully developed turbulent and two dimensional, the thermal conductivity of the channel wall and chevron material do not change with temperature, and the channel wall and chevron material are homogeneous and isotropic with an enhanced wall treatment function. The solutions are considered to be converged when 
the normalized residual values are less than $10^{-6}$ for all variables, but less than $10^{-5}$ only for the continuity equation.

A grid independence test has been performed for the channel to analyze the effects of grid sizes on the results, as shown in Fig. 3. It is found that further increase in the grid beyond 51159 cells results in a variation in the Nusselt number less than $1 \%$, thus this grid number is taken as a criterion of grid independence. This fine mesh size is able to provide good spatial resolution for the distribution of most variables within the channel.

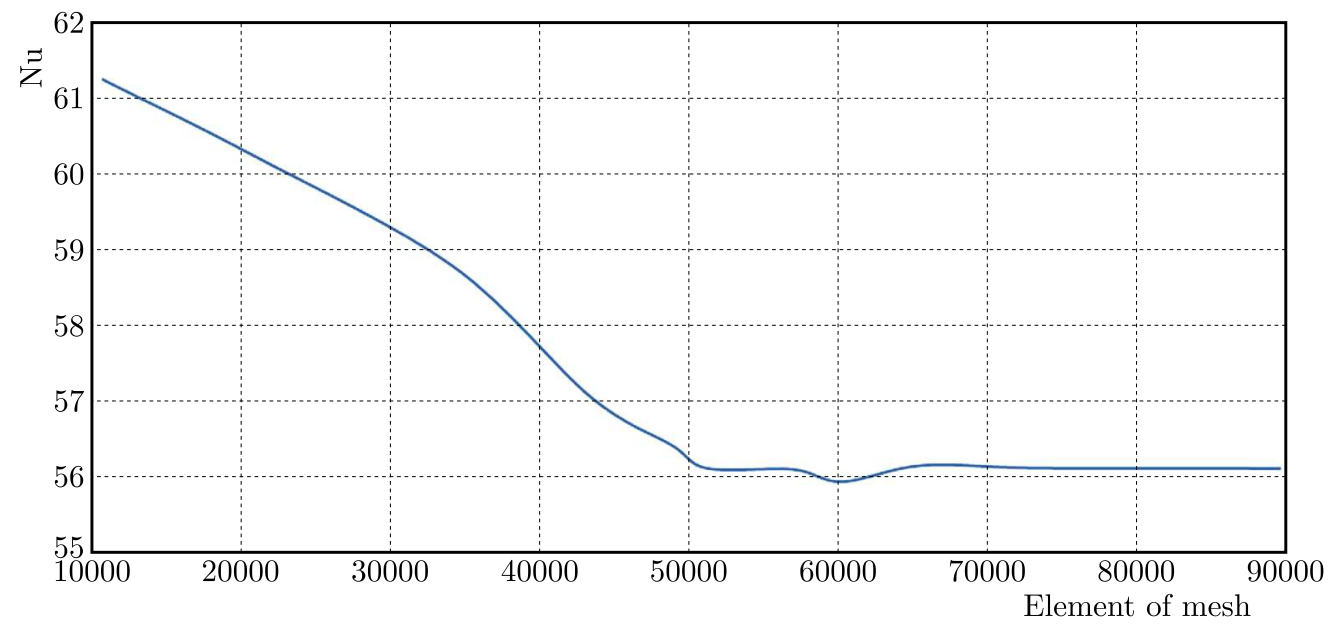

Fig. 3. Investigation of the independency of mesh elements

Figure 4 shows the grid size of mesh elements near the chevron walls. To investigate the independency of the mesh, the number of mesh elements increases near the chevron walls by $y^{+}=2.014253$.

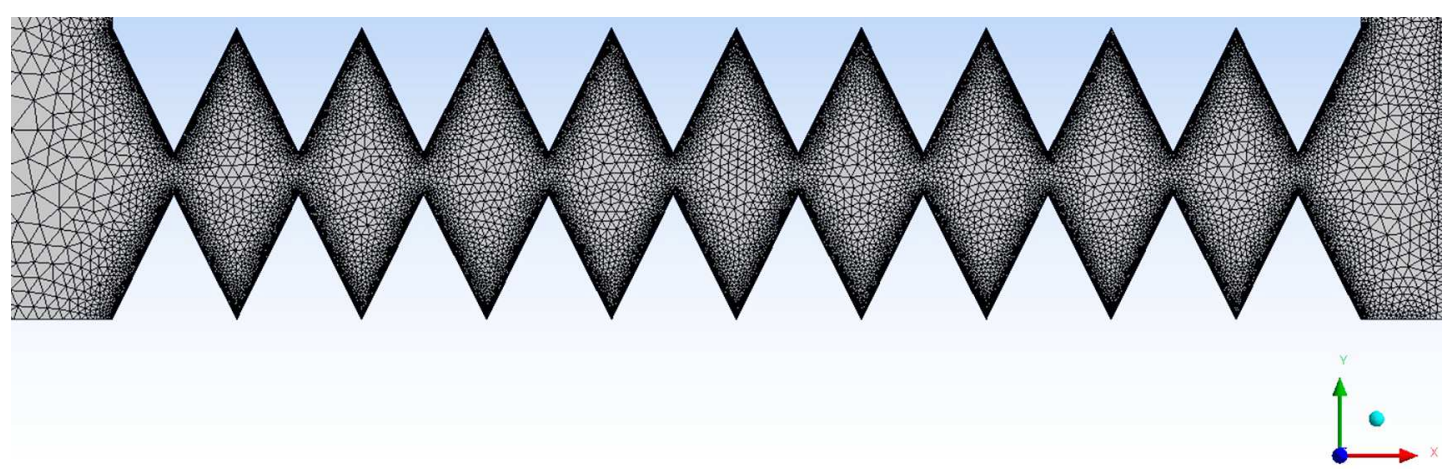

Fig. 4. Grid size of mesh element near the chevron walls

\section{Results and discussion}

\subsection{Phase shift and Reynolds numbers variation in a triangular chevron channel}

Figure 5 shows experimental results of temperature variation of the fluid vs. dimensionless distance $x / D$ for a phase shift of $0^{\circ}$ and $D=5 \mathrm{~mm}$. The air temperature has increased by an increase in the dimensionless distance. The same results are for other phase differences.

Figure 6 illustrates the average fluid temperature across the triangular chevron wall for different Reynolds numbers. The fluid temperature significantly decreases with the increasing Reynolds number. The difference between the numerical and experimental results is about $1 \% \leqslant$ error $\leqslant 11 \%$, which proves good validation between both results. 


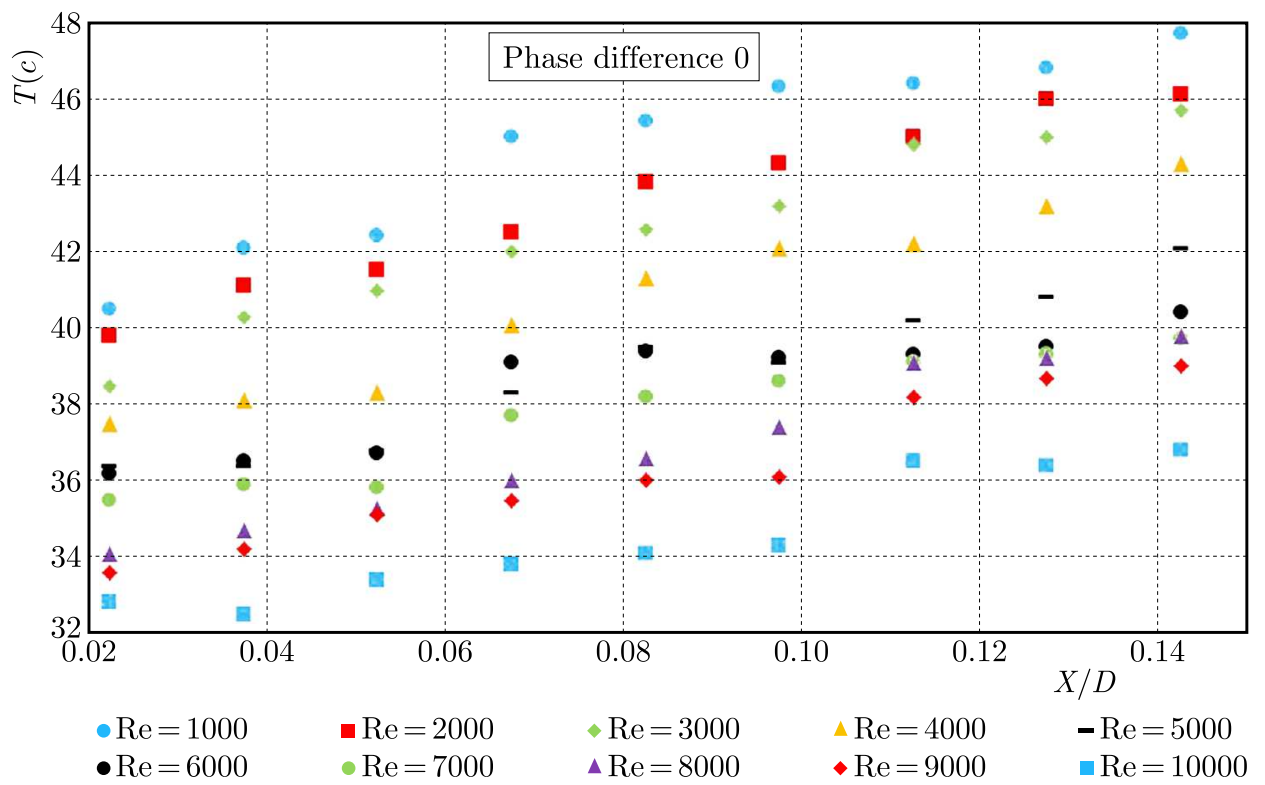

Fig. 5. Experimental results of temperature variation of the fluid vs. dimensionless distance $x / D$ for a phase shift of $0^{\circ}$ and $D=5 \mathrm{~mm}$

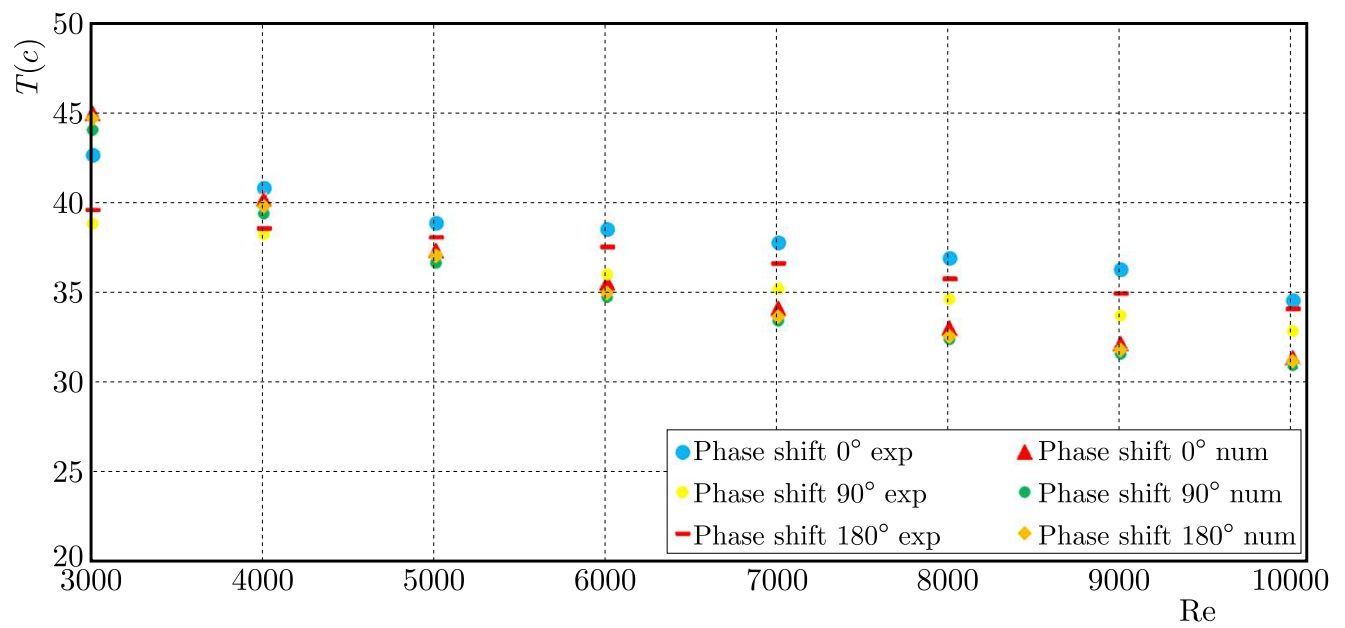

Fig. 6. Variation and validation of the average temperature of the fluid vs. Re in experimental and numerical results at phase shifts $0^{\circ}, 90^{\circ}, 180^{\circ}$

The numerical and experimental results of the variation of $\mathrm{Nu}$ for three phase shifts and within $3000 \leqslant$ Re $\leqslant 10000$ are shown in Fig. 7. The Nusselt number for the chevron channels is higher than for the plain channel because the Nusselt number depends on the heat transfer rate. On the other hand, the values of the Nusselt number are found to increase with an increase in Re in all cases. Best results are obtained for the phase difference $90^{\circ}$. The Nusselt number for $\phi=90^{\circ}$ is about $4.8 \%$ higher than for $\phi=0$. In fact, $\phi=90^{\circ}$ makes more turbulence interrupts in the development of a thermal boundary layer. The vortices induced in and around the chevron channels are thought to be responsible for the increase of turbulence intensity of the flow which leads to higher heat transfer rates.

Figure 8 shows the experimental and numerical results of the effect of the Reynolds number and the relative phase shift on the friction factor. When the fluid passes through the chevron channel, a considerable pressure drop occurs. The value of the friction factor decreases with the increasing Reynolds number in all cases, as expected, due to suppression of the viscous sub-layer with an increase in Re. Also, the pressure drop in the channel with a phase shift $\phi=180^{\circ}$ is 


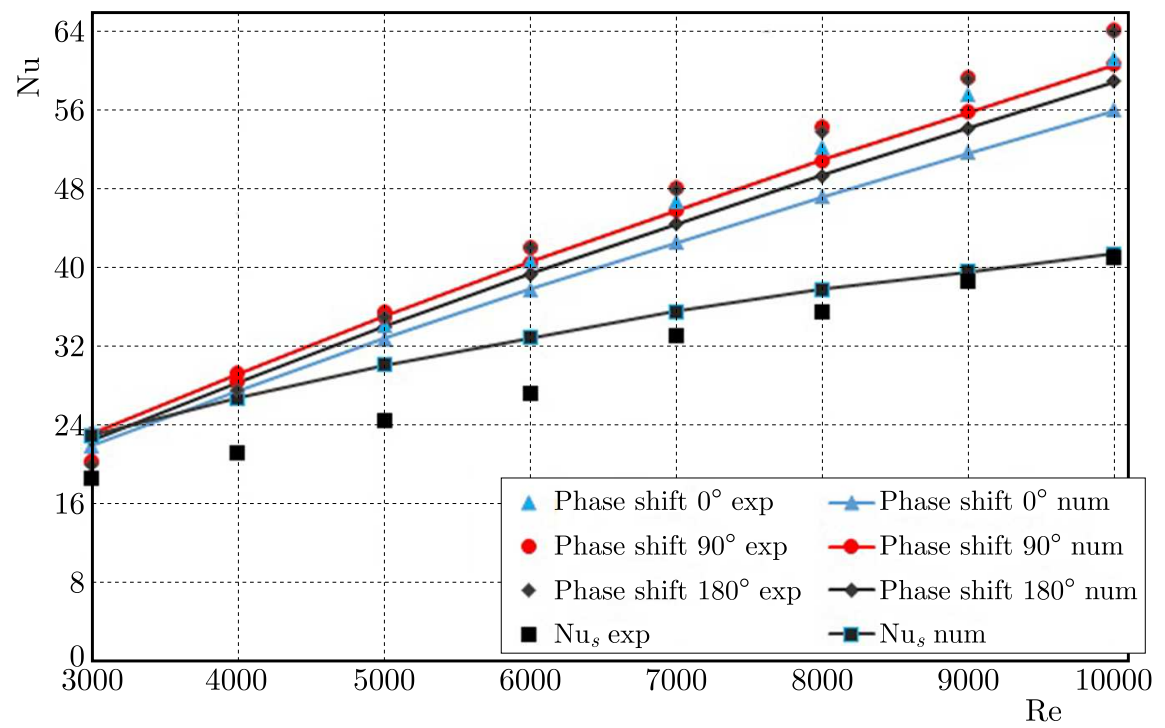

Fig. 7. Variation of $\mathrm{Nu}$ vs. Re for phase shifts $0^{\circ}, 90^{\circ}, 180^{\circ}$ in experimental and numerical results

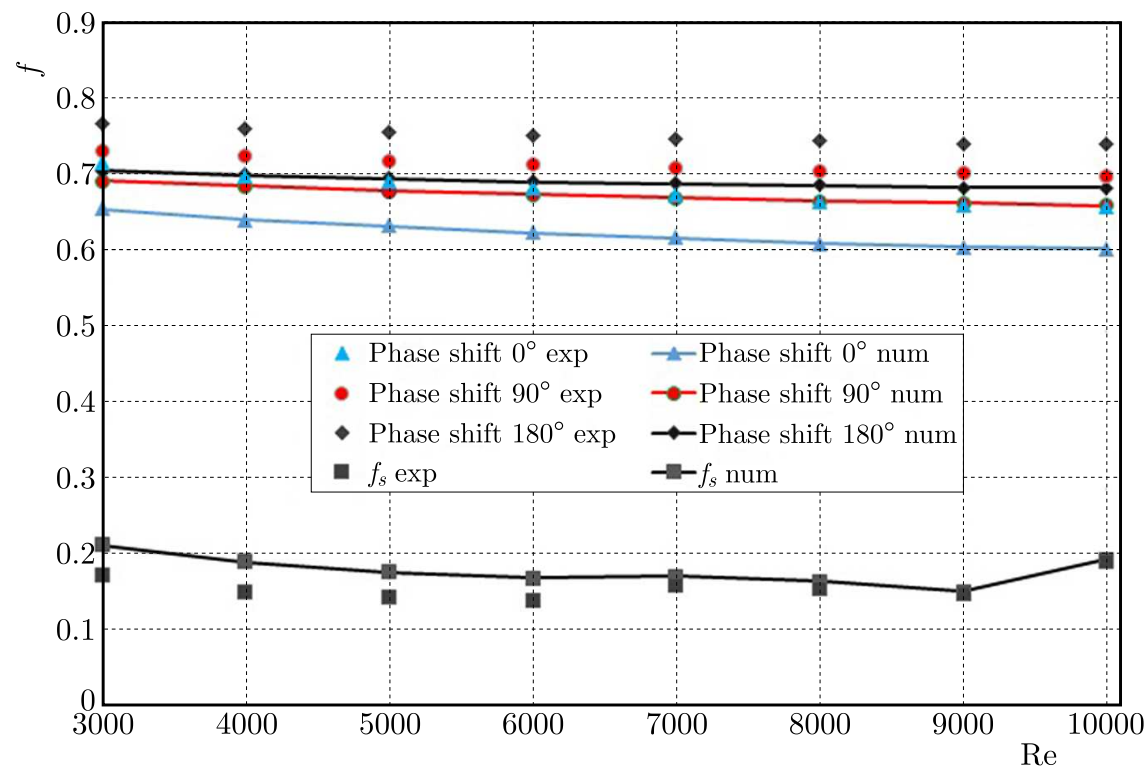

Fig. 8. Variation of $f$ vs. Re for phase shifts $0^{\circ}, 90^{\circ}, 180^{\circ}$ in experimental and numerical results

greater than in other cases. As expected, the $f$ from the $\phi=180^{\circ}$ array is substantially higher than that with $\phi=0^{\circ}$ and $\phi=90^{\circ}$, whereas the $\phi=0^{\circ}$ yields the lowest $\phi$. The $f$ value of $\phi=180^{\circ}$ is found to be about $4 \%$ above $\phi=90^{\circ}$ and about $5.4 \%$ over the $\phi=0^{\circ}$. It can be interpreted that the $\phi=180^{\circ}$ causes a higher turbulence intensity in the flow due to more oscillating streamlines than for the other phase shifts (see Fig. 10). But it creates a "stronger" recirculation region and vortices inside the chevron surfaces, so it prevents the fluid from good mixing. Thus, it results in a less increase in the heat transfer in comparison with both $\phi=90^{\circ}$ and $\phi=180^{\circ}$. Using the phase shift, one can reduce the occurrence of the recirculation providing more surface sweep, therefore, the negative effect of the recirculation region on the heat transfer can be decreased. The shift between the numerical and experimental results for each phase shift is about $1 \% \leqslant$ error $\leqslant 11 \%$, which proves a good validation.

Figure 9 shows the variation of TEF with Re for all phase shifts. The combined effect of $\mathrm{Nu}$ and $f$ values has been simplified by TEF, Eq. (3.8). In general, TEF tends to decrease with the 
rise of Re. It is worth noting that the TEF value of the phase shift 90 is the highest and found to be the best among other phase shifts.

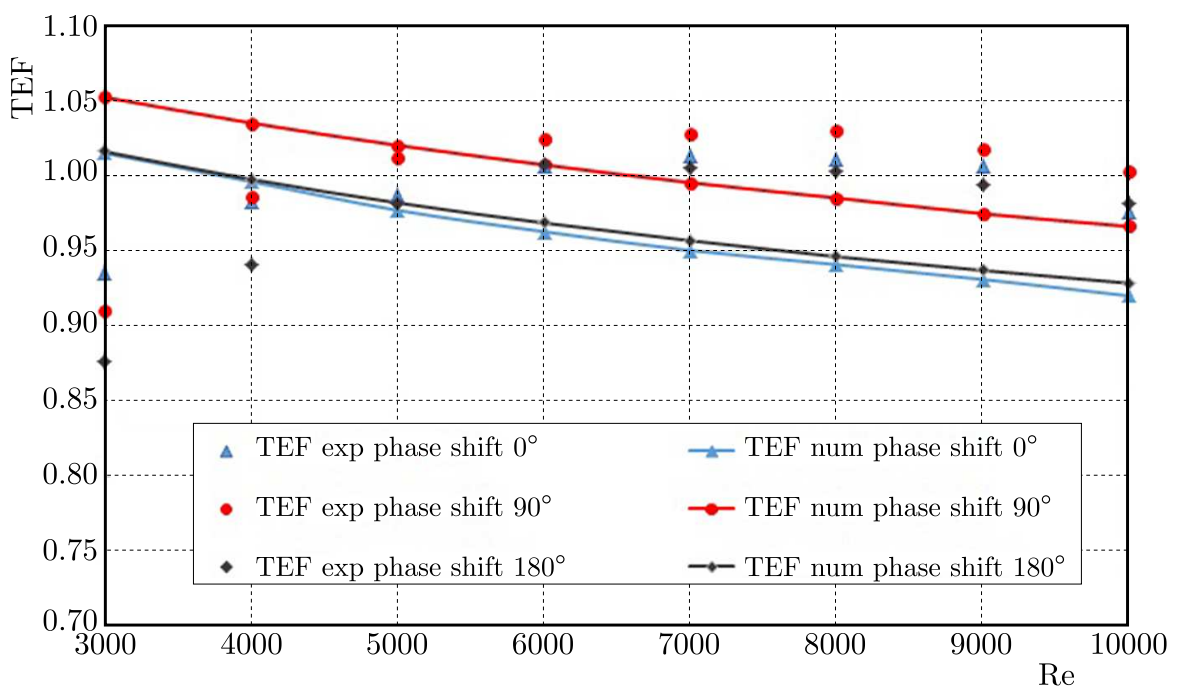

Fig. 9. Variation of TEF vs. Re for phase shifts $0^{\circ}, 90^{\circ}, 180^{\circ}$ in experimental and numerical results

For phase shifts $0^{\circ}, 90^{\circ}$ and $180^{\circ}$, the maximum TEF values are, respectively, about $1.05,1.1$ and 1.07. It is seen that the phase shift $90^{\circ}$ gives the highest TEF at lower Reynolds numbers. At the given chevron surfaces, the phase shift $90^{\circ}$ yields TEF around $3-7 \%$ higher than that of other two phase shifts. Because of considerably higher $\mathrm{Nu}$ and lower $f$, only for the phase shift $90^{\circ}$ will be further investigated in the subsequent Section.

The variation of the dimensionless parameters $\mathrm{Nu}, f$ and TEF versus Re for phase shifts $0^{\circ}$, $90^{\circ}$ and $180^{\circ}$ are presented in Figs. 7, 8 and 9, respectively. For three cases and at all positions, it is observed that there is a relatively good concordance between the experimental data and the numerical results. For very low flow rates, as in the experiment, it is believed that the observed differences between the experimental and numerical data may be partly caused by a small error in the DC source power, heater and the manometer velocity sensor in the system, which can slightly disrupt the turbulence flow. Another possible cause of these differences is the fact that the hot junction of the thermocouples, which are the top of the plate surface, covers a small circular area with a diameter of approximately $1 \mathrm{~mm}$ and, therefore, the measured temperature is actually the average temperature of that small surface.

Figure 10 shows the streamlines associated by a modified various phase shift at $\operatorname{Re}=10000$. By focus on the upstream flow structures of various phase shifts, the phase shift is $\phi=90^{\circ}$ and the size of bubbles decrease. The volume of the bubbles, induce larger recirculation zones which provide higher turbulent intensity. This may be related to a decrease of the laminar sub-layer and to the maximum heat transfer for $\phi=90^{\circ}$. When the phase shift is close $\phi=90^{\circ}$, it creates more vortex and decreases bubbles from triangular chevron surfaces. When the phase shift angle increases, the friction factor also increases because the fluid is oscillating between chevron surfaces. It is clear that the effect of the phase shift on temperature and flow development in the chevron channel is that there are smaller recirculation regions and more separation bubble regions formed in the adjacent inlet/outlet. This effect increases by closing to the phase shift $\phi=90^{\circ}$, which means that the influence of the wall on the main stream becomes greater. This generates greater swirl flow in the wavy wall due to transfer vortices of the bulk flow field in the wavy wall. This induces a higher temperature gradient near the wavy wall. Therefore, the net heat transfer rate from the wavy wall to the fluid is increased. 

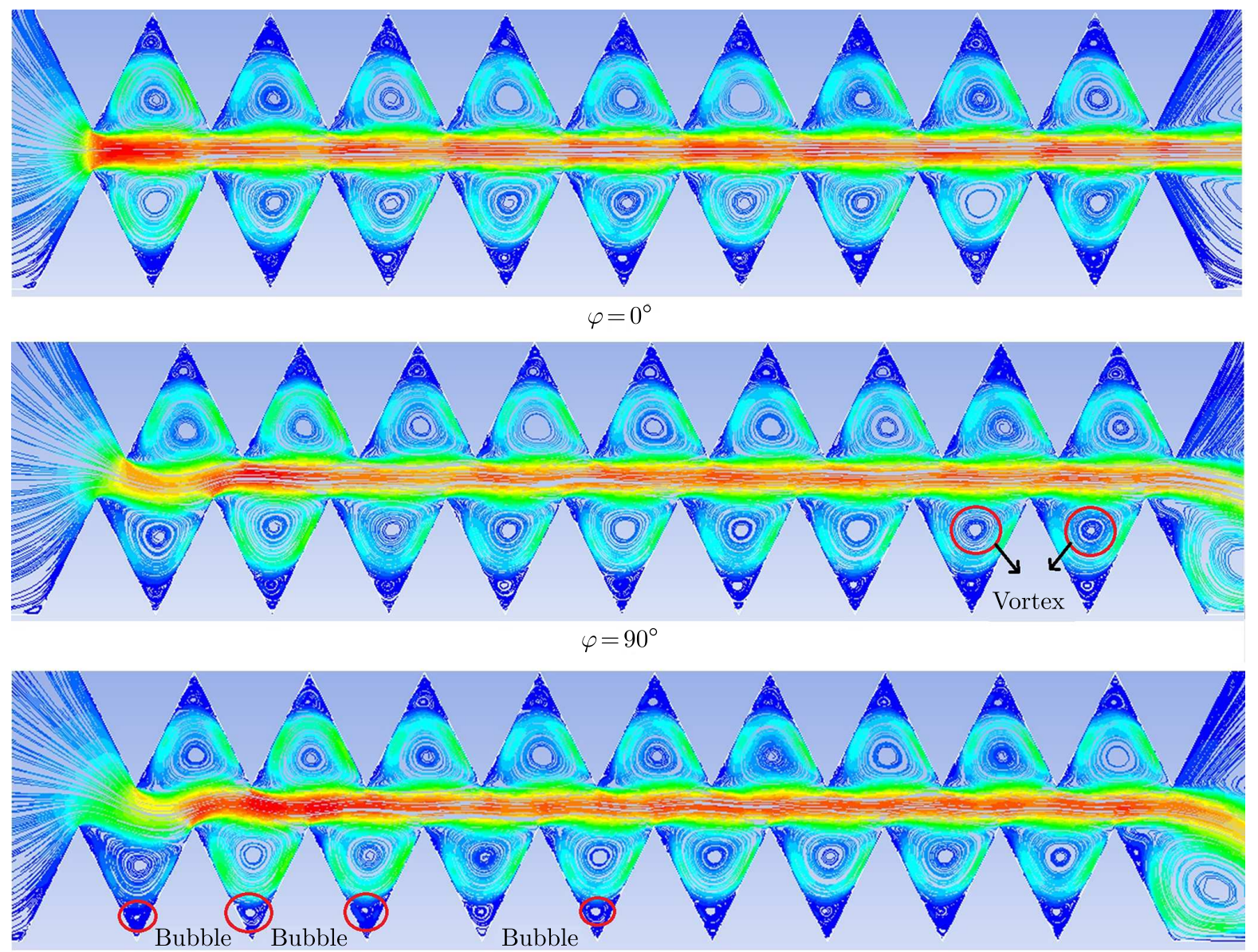

$\varphi=180^{\circ}$

Fig. 10. Streamline of fluids for phase shifts $0^{\circ}, 90^{\circ}, 180^{\circ}$

\subsection{Effect of distance between triangular chevron surfaces}

Figure 11 demonstrates the variation of the average fluid temperature above $7.5 \mathrm{~mm}$ from the triangular chevron plate according to numerical and experimental results. In Fig. 11, it is seen that the numerical calculations with the standard wall function are similar to the experimental data with the chevron channel for $\operatorname{Re}=10000$ and $\phi=180^{\circ}$. The wall temperature increases when the distance between the surfaces increases. It can interpreted that the fluid flow becomes more complex near the wall region when $D$ increases. As a result, the velocity of fluids decrease with groving $D$ and the temperature near the wall becomes warmer.

The average numerical and experimental Nusselt number for triangular chevron surfaces at $\operatorname{Re}=10000$ for $\phi=180^{\circ}$ is presented in Fig. 12. There is good agreement between numerical and experimental results with an error less than $10 \%$ for most of the results. It can be seen that with an increase in the distance between triangular chevron surfaces, the Nusselt number decreases. In fact, the volume of vortices in the laminar sublayer and the velocity of fluids decrease. Figure 12 shows $\mathrm{Nu}$ for triangular chevron surfaces. It is greater than for a smooth surface below $D=15 \mathrm{~mm}$. When $D$ increases for a constant $\mathrm{Re}=10000$, the velocity of the fluid decreases. As a result, $\mathrm{Nu}$ for triangular chevron surfaces is less than that for smooth surfaces above $D=15 \mathrm{~mm}$.

Figure 13 shows the variation of the experimental and numerical friction factor along the channel for $\operatorname{Re}=10000$ and $\phi=180^{\circ}$. There is a good validation of the numerical and experimental results with an error less than $9 \%$ for most of results. The friction factor for triangular 


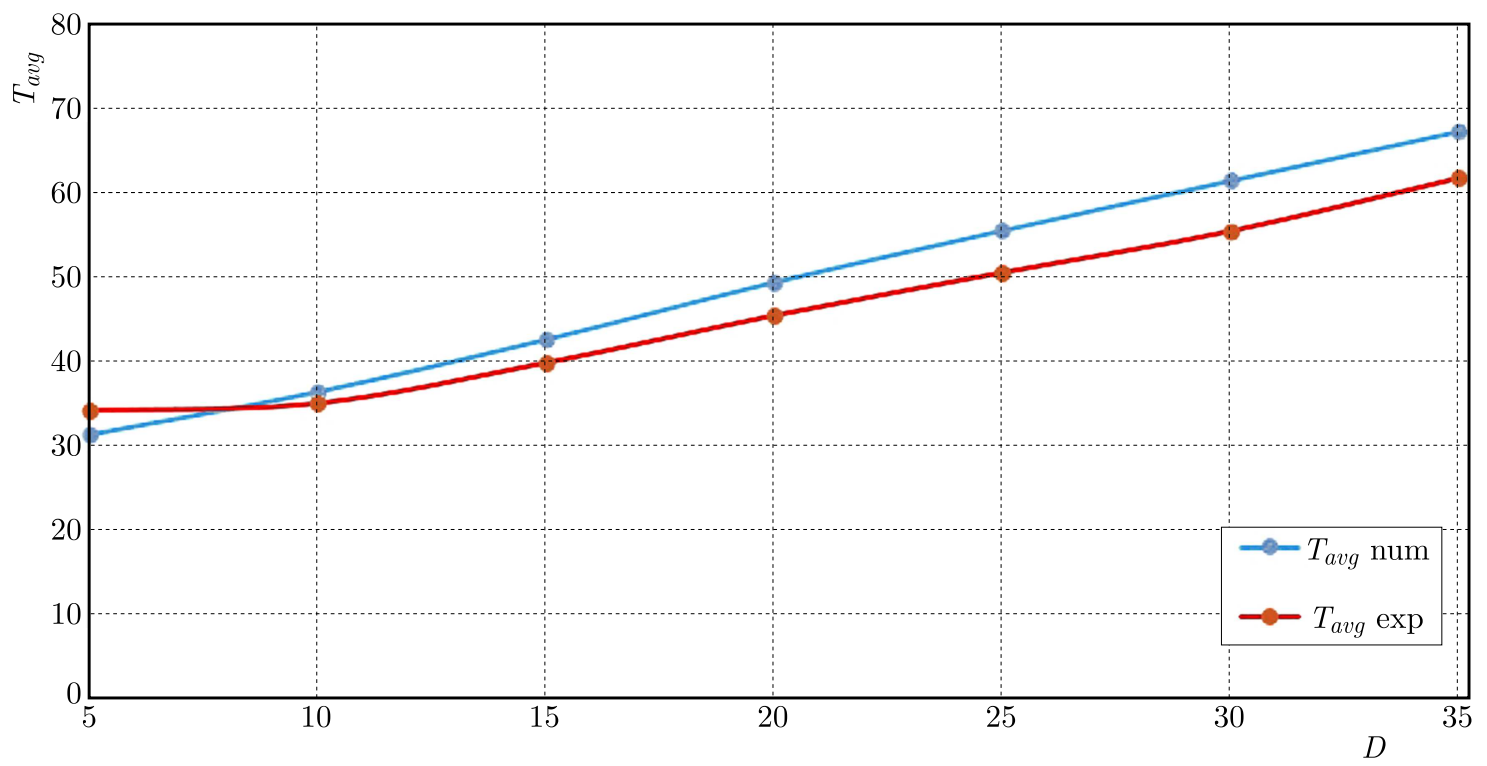

Fig. 11. Experimental and numerical variation of average temperature of the chevron plate vs. different distances

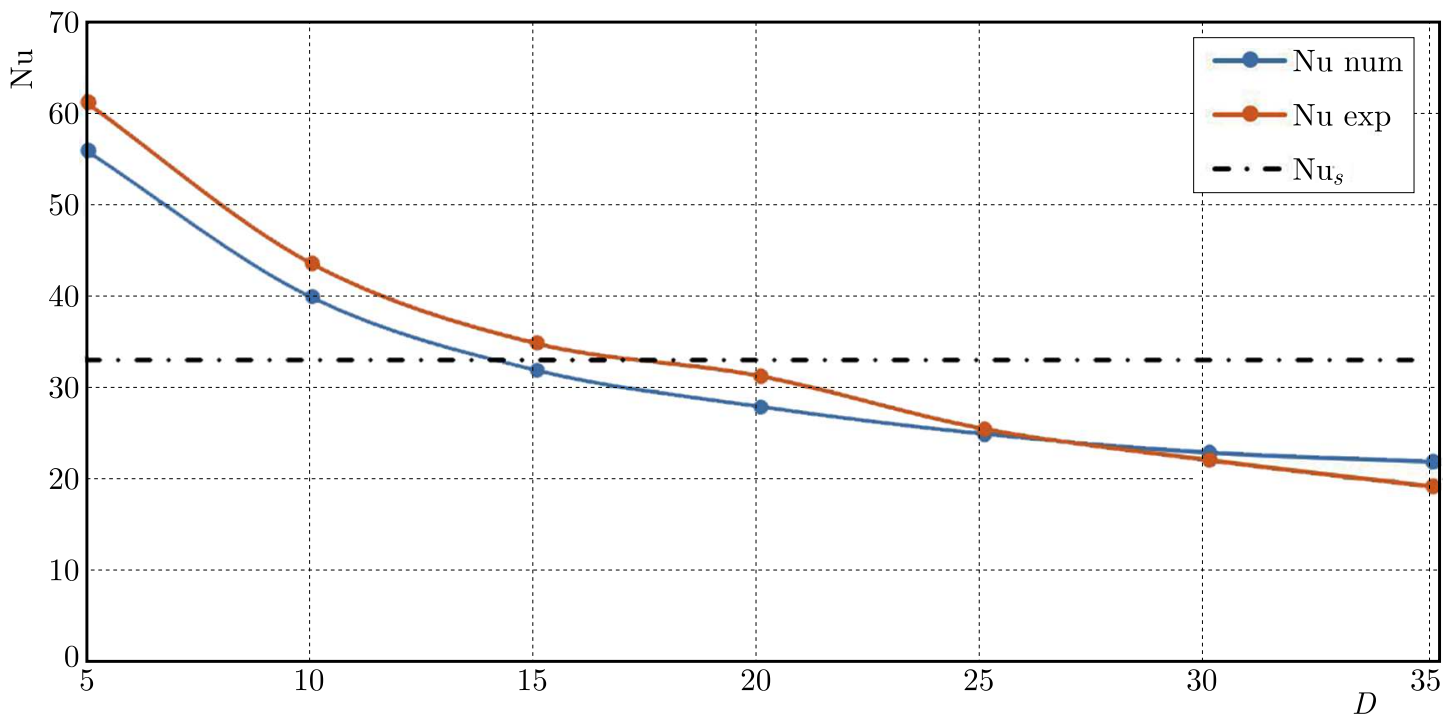

Fig. 12. Experimental and numerical variation of $\mathrm{Nu}$ of the chevron plate vs. different distances

chevron surfaces is the highest compared to the smooth surface. It is obvious that the friction factor decreases gradually for all configurations with an increase in the Reynolds number.

Based on the same pumping power consumption, the TEF is shown and compared for differently distanced chevron surfaces tested, see Fig. 14. By considering the heat transfer and the pressure drop simultaneously at $\operatorname{Re}=10000$ and $\phi=180^{\circ}$, the working conditions should give a high TEF. It is seen from the figure that the TEF tends to decrease as $D$ increases (for numerical results). Consistently, the investigation reveals a higher thermal enhancement factor at $D=5 \mathrm{~mm}$. There appears transfer of additional heat by conduction which provides a better fluid mixing. As a result, to obtain the maximum TEF, one should to get the heat exchanger with the minimum distance between surfaces, see Fig. 14. 


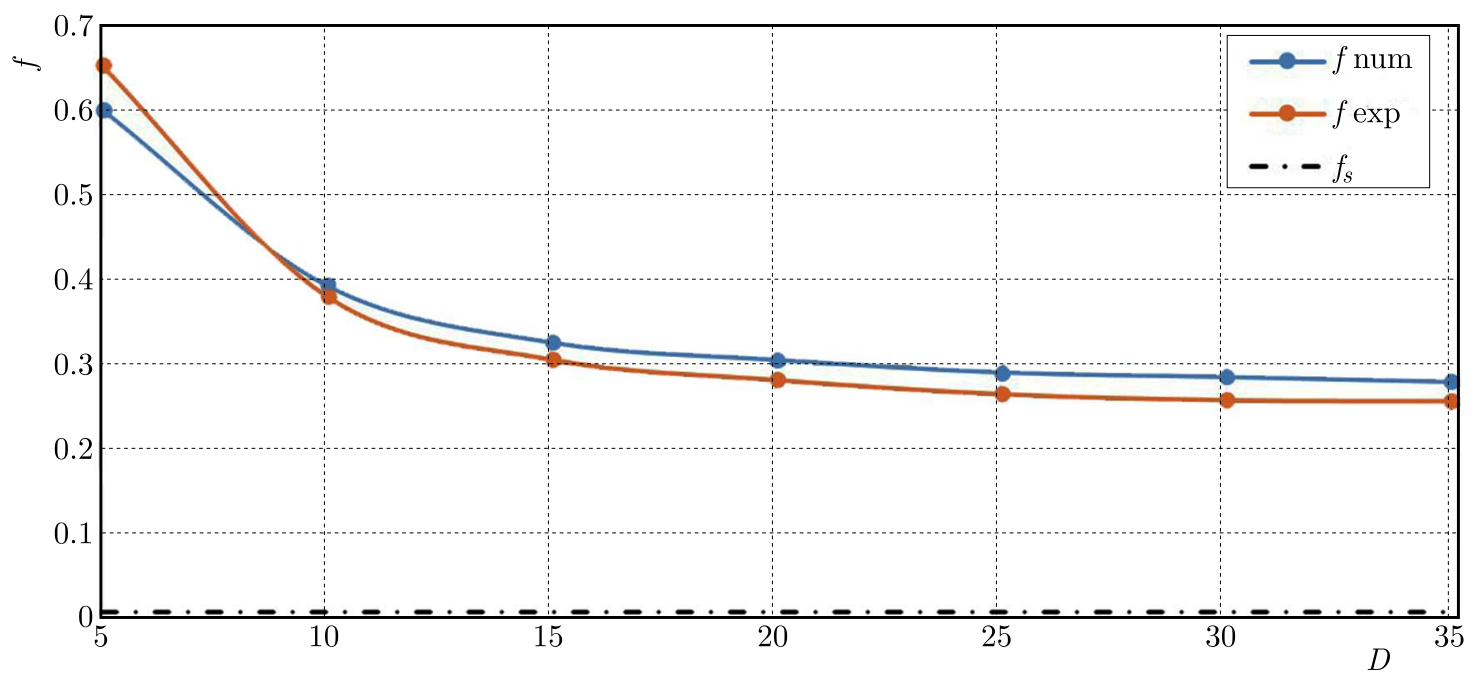

Fig. 13. Experimental and numerical variation of the friction factor for the chevron plate vs. different distances

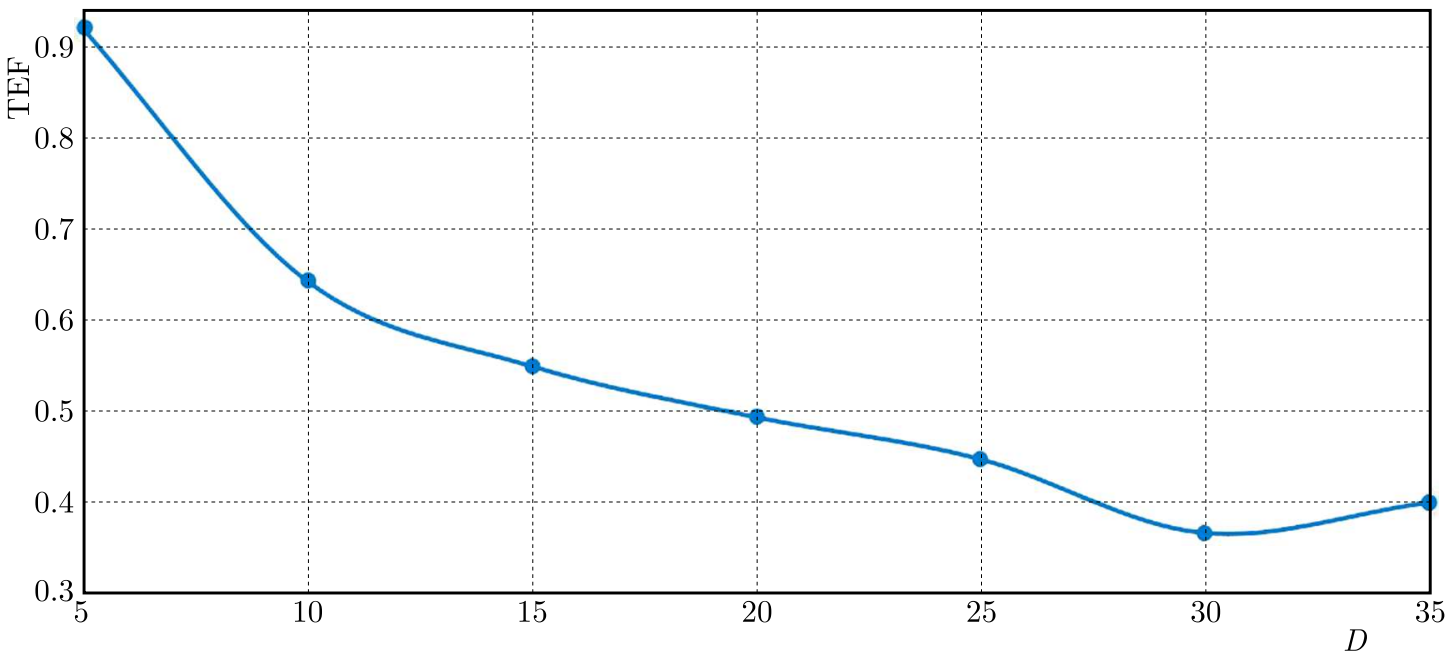

Fig. 14. Variation of TEF for the chevron plate vs. different distances

\section{Conclusions}

A triangular chevron channel is a good alternative in high heat flux applications or more efficient heat exchange devices used in a variety of engineering structures such as heating and air conditioning systems. In this paper, the effect of phase shift and distance between chevron channels is examined for Reynolds numbers ranging from 1000 to 10000 . The aim of the stugy is to achieve to the maximum heat transfer and thermal enhancement factor as well as the minimum pressure drop. The best phase shift angle is $\phi=90^{\circ}$. Also the distance between chevron surfaces $D$ has been investigated. The results show that $D=5 \mathrm{~mm}$ is the best distance between chevron surfaces in getting the maximum TEF and $\mathrm{Nu}$, and the minimum $f$. Decreasing and holding the average base temperature of the chevron surfaces at a constant level, particularly at higher Reynolds numbers has been successfully achieved. Increasing the Reynolds number leads to a more complex fluid flow and the heat transfer rate. When the phase shift gets close to $\phi=90^{\circ}$, $\mathrm{Nu}$ and TEF reach the maximum rate, whereas $f$ the minimum nalue. The channels with the phase shift angle $\phi=90^{\circ}$ are the most attractive from the viewpoint of energy saving compared to others with the phase shifts $\phi=0^{\circ}$ and $180^{\circ}$. For the triangular chevron surfaces, the 
maximum heat transfer is obtained from $33.33 \%$ up to $37.5 \%$, which is higher than for smooth surfaces.

\section{References}

1. Al-Shamani A.N., Sopian K., Mohammed H.A., Mat S., Ruslan M.H., Abed A.M., 2015, Enhancement heat transfer characteristics in the channel with Trapezoidal rib-groove using nano fluids, Case Studies in Thermal Engineering, 5, 48-58

2. Dellil A., Azzi A., Jubran B.A., 2004, Turbulent flow and convective heat transfer in a chevron wall channel, Heat and Mass Transfer, 40, 793-799

3. Eiamsa-ard S., Changcharoen W., 2011, Analysis of turbulent heat transfer and fluid flow in channels with various ribbed internal surfaces, Journal of Thermal Science, 20, 3

4. Islamoglu Y., Parmaksizoglu C., 2004, Numerical investigation of convective heat transfer and pressure drop in a chevron heat exchanger channel, Applied Thermal Engineering, 24

5. Khoshvaght-Aliabadi M., Sahamiyan M., Hesampour M., Sartipzadeh O., 2016, Experimental study on cooling performance of sinusoidal-chevron minichannel heat sink, Applied Thermal Engineering, 92, 50-61

6. Moon M.-A., Park M.-J., Kom K.-Y., 2014, Evaluation of heat transfer performances of various rib shapes, International Journal of Heat and Mass Transfer, 71, 275-284

7. SAKR M., 2015, Convective heat transfer and pressure drop in V-chevron channel with different phase shifts, Heat Mass Transfer, 51, 129-141

8. Vanaki Sh.M., Mohammed H.A., Abdollahi A., Wahid M.A., 2014, Effect of nanoparticle shapes on the heat transfer enhancement in a chevron channel with different phase shift, Journal of Molecular Liquids, 196, 32-42

9. Xie G., Liu J., Ligrani P.M., Sunden B., 2014, Flow structure and heat transfer in a square passage with offset mid-truncated ribs, International Journal of Heat and Mass Transfer, 71, 44-56

10. YAng Y.-T., Chen P.-J., 2010, Numerical simulation of fluid flow and heat transfer characteristics in channel with V chevron plates, Heat Mass Transfer, 46, 437-445

11. Yongsiri K.A., Eiamsa-ard P.B., Wongcharee K.C, Eiamsa-Ard S., 2014, Augmented heat transfer in a turbulent channel flow with inclined detached-ribs, Case Studies in Thermal Engineering, 3, 110 